\title{
External Beam Radiotherapy only as an Alternative for Radical Cystectomy in Highly Selected Bladder Cancer Patients
}

\author{
Valérie Fonteyne $^{1 *}$, Tom Vercauteren ${ }^{1}$, Nicolaas Lumen ${ }^{2}$, Werner De Gersem ${ }^{1}$, Bruno Speleers ${ }^{1}$, Geert \\ Villeirs $^{3}$, Piet Ost ${ }^{1}$, Pieter Devisschere ${ }^{3}$ and Karel Decaestecker ${ }^{2}$ \\ ${ }^{1}$ Departement of radiotherapy-oncology, Ghent University Hospital, Belgium \\ ${ }^{2}$ Departement of urology, Ghent University Hospital, Belgium
}

${ }^{3}$ Departement of radiology, Ghent University Hospital, Belgium

*Corresponding author: Valérie Fonteyne, Department of Radiotherapy-Oncology, Ghent University Hospital, Belgium

\section{ARTICLE INFO}

Received: 㟒 April 08, 2019

Published: 慧 April 17, 2019

Citation: Valérie F, Tom V, Nicolaas L, Werner D G, Bruno S. External Beam Radiotherapy only as an Alternative for Radical Cystectomy in Highly Selected Bladder Cancer Patients. Biomed J Sci \& Tech Res 17(1)-2019. BJSTR. MS.ID.002956.

Abbreviations: ePLND: extended Pelvic Lymph Node Dissection; EBRT: External Beam Radiotherapy; TURb: Transurethral Resection of the Bladder; mp-MRI: Multiparametric Magnetic Resonance Imaging
ABSTRACT

Background: Bladder sparing therapies are an alternative for Radical Cystectomy (RC) for patients with Muscle Invasive Bladder Cancer (MIBC). Addition of chemotherapy to External Beam Radiotherapy (EBRT) results in increased toxicity. In properly selected patients, re-evaluating the place of EBRT alone might be of interest.

Methods: A phase 1 trial was initiated for highly selected MIBC patients. Only patients with histological diagnosis of MIBC and stage <T3bN0M0 were eligible. After complete resection of the Gross Tumor Volume (GTV) and negative node status on Pelvic Lymph Node Dissection (PLND), EBRT upto 40 Gy in 20 fractions was administered to the whole bladder with a simultaneous integrated boost (SIB) to the GTV according to 3 dose levels:

a) Level 1: 57.6 Gy (20 x 2.88 Gy, 5 fractions/week, 4 weeks); BED: 61 Gy.

b) Level 2: 60 Gy (20 x 3.00 Gy, 5 fractions/week, 4 weeks); BED: 64 Gy

c) Level 3: 62.4 Gy (20 x 3.12Gy, 5 fractions/week, 4 weeks); BED: 67 Gy.

For all levels: BED was calculated with $\otimes / \otimes=13$.

A $3+3$ design was adopted. Once 1 dose level was proven to be safe (i.e. no acute RTOG $>$ Grade3 urinary toxicity) patients were treated up to a higher dose level. Treatment was performed with volumetric arc therapy. A plan of day the approach was applied.

Results: The trial was prematurely closed due to poor recruitment after inclusion of only 3 patients. One patient received a RC due to pN1 status after PLND. The other 2 patients were treated up to 57.6 Gy to the GTV. Cystoscopic evaluation revealed no signs of recurrence after 39 and 25 months. No grade 4 toxicity was reported. Rectal toxicity was limited to grade 1 toxicity. Acute grade 3 urinary toxicity was observed in both patients. At latest follow up, urinary toxicity was limited to grade 2 nocturia and grade 2 retention.

Conclusion: No major toxicity was observed and oncological outcome is excellent with more then 2 years of follow up after EBRT alone for MIBC. These preliminary results might be considered as hypothesis generating for developing studies with high dose EBRT in well-selected MIBC patients.

\section{Introduction}

The gold standard for patients with Muscle Invasive Bladder Cancer (MIBC) is a Radical Cystectomy (RC) and extended Pelvic Lymph Node Dissection (ePLND). Peri-operative mortality rate is \pm $2 \%$ and major toxicity is reported in up to $30 \%$ of the patients [1]. To increase quality of life, bladder-sparing trimodality therapies
(BST) (i.e Transurethral Resection of the Bladder (TURb) + EBRT + chemotherapy) are considered a valuable alternative for RC for MIBC patients with similar local control and cause specific survival rates as reported in modern surgical series [2,3]. But also, with BST grade $\geq 3$ toxicity has been reported in up to $36 \%$ of the patients [4]. Omitting chemotherapy reduces the risk of developing 
severe gastro-intestinal, urinary and haematological toxicity at the cost of worse disease-free survival [4]. In properly selected patients, re-evaluating the place of radiotherapy alone might be of interest. Therefore we initiated a phase 1 trial in which EBRT with a hypofractionated Simultaneous Integrated Boost (SIB) to the original tumor localisation was administered after complete resection of the lesion in node negative MIBC patients (Figure 1).

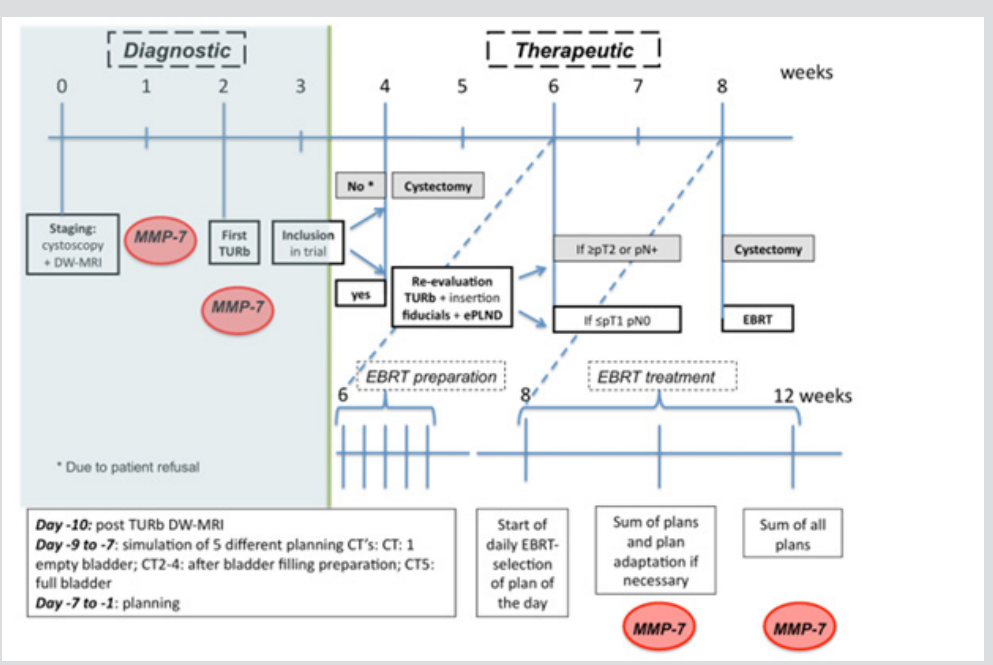

Note: Within the protocol matrix metalloproteinase's-7 (MMPs) was determined in the blood and urine as a biomarker.

Figure 1: Flow chart of the treatment protocol.

\section{Material and Methods}

We designed a phase 1 trial to evaluate the place of EBRT only in highly selected MIBC patients. The ethic committee of Ghent University Hospital approved the study on 10/03/2015. The study was registered on Clinicaltrials.gov (NCT02748200). Eligible patients were patients with histological proven diagnosis of MIBC, initial stage $<$ T3b tumours, no distant metastasis, WHO performance state $0-2$, age $\geq 18$ and $<80$ years, signed informed consent and no contra-indication for diffusion-weighted magnetic resonance imaging (DW-MRI). Before being referred for EBRT, patients underwent a second Transurethral Resection of the Bladder (TURb) in order to obtain a complete resection (defined as $\leq \mathrm{pT} 1$ at re-TURb) as well as an ePLND to exclude pathologically involved lymph nodes. At that time gold markers 0,4 x 5 mm (QLRAD, Netherlands) were inserted at the edges of the TURb region.

For each patient 5 different planningsCT's were performed with 5 different bladder volumes following a standardized protocol. The clinical target volume consisted of the whole bladder and was defined on each planningsCT separately. The whole bladder was treated to 40 Gy in 20 fractions, 5 fractions/week, 4 weeks in total. Based on the summation of abnormalities seen on pre(initial tumor region) and post TURb (zone of fibrosis) DW-MRI images, the tumor region was delineated and defined as a Gross Tumor Volume (GTV). The GTV was treated with a simultaneous integrated boost (SIB), without extending the 4-weeks treatment period. Three different dose levels to the GTV were implemented in order to increase the biological equivalent dose (BED), as MIBC has been shown to be dose-sensitive [6]. A 3+3 design was adopted. Once 1 dose level was proven to be safe (i.e. no acute RTOG $>$ Grade 3 urinary toxicity) patients were treated up to a higher dose level.

Dose prescription on $50 \%$ of the GTV was: a) Level 1: 57.6 Gy (20 x 2.88 Gy, 5 fractions/week, 4 weeks); BED: 61 Gy.

b) Level 2: 60 Gy (20 x 3.00 Gy, 5 fractions/week, 4 weeks); BED: $64 \mathrm{~Gy}$

c) Level 3: 62.4 Gy ( 20 x 3.12Gy, 5 fractions/week, 4 weeks); BED: 67 Gy. For all levels: BED was calculated with $\alpha / \beta=13$ (5).

The volume of the rectum and sigmoid receiving 34.3 Gy and 34.4 Gy were constrained to $64 \%$ and $35 \%$, to avoid grade $\geq 2$ rectal toxicity with a probability of $90 \%$ and $95 \%$ respectively [7]. The dose received by $2 \%$ of the volume, considered as a surrogate for the maximum dose, was limited to 40 Gy for the femoral heads, small intestine and symphysis. Two sets of 5 treatment plans were generated. For one set, 5 treatment plans were optimized individually. An accumulated dose distribution was created on the third planning CT data set, with a comfortably filled bladder, using the deformation fields of deformable image registration. The other set of treatment plans were optimized simultaneously using Multiple Plan Optimization (MPO). MPO enables the specification of dose objectives on individual treatment plans and on the accumulated dose distributions. Treatment was delivered on an Elekta 18-MV linear accelerator (LINAC, Crawley, UK) with Volumetric Arc Therapy (VMAT) technique.

A daily cone beam CT was performed in order to evaluate bladder filling. After matching with the different planning CT's the most appropriate planning was selected. After 10 sessions an accumulated dose was calculated based on the delivered plans. Considering the dose that was already delivered in daily practice during the first 10 sessions, new plans were created. In order to do so, the planning procedure was repeated for the last 10 fractions. Patients were followed weekly during therapy, 1 month after 
therapy and 3- monthly thereafter. At each follow up after EBRT a standard blood control was performed. In asymptomatic patients imaging (cystopscopy of the pelvis and CT thorax/abdomen) was performed 3-monthly during the first year and 6-monthly thereafter up to a period of 5 years or until progression. A DW-MRI was performed at least once after the end of radiotherapy.

The primary outcome is acute RTOG $\geq$ Grade3 toxicity within

\section{Results}

Table 1: Patient characteristics and treatment details.

\begin{tabular}{|c|c|c|c|c|c|}
\hline Characteristics & & & Patient 1 & Patient 2 & Patient 3 \\
\hline Age at diagnosis (year) & & & 78 & 65 & 74 \\
\hline \multirow{4}{*}{ Tumor characteristics } & \multirow{2}{*}{\multicolumn{2}{|c|}{ Oncological antecedents }} & $\begin{array}{c}\text { 2013: pTa G2 TCC treated with } \\
\text { TURb }\end{array}$ & \multirow{2}{*}{$\begin{array}{l}\text { 2016: initial diagnosis of } \\
\text { pT2 G3 TCC }\end{array}$} & \multirow{2}{*}{$\begin{array}{l}\text { 2016: initial diagnosis } \\
\text { of pT2 G3 TCC }\end{array}$} \\
\hline & & & $\begin{array}{l}\text { 2015: recurrent pT2 G3 TCC: } \\
\text { start of Mitomycine } 4 \text { cycles }\end{array}$ & & \\
\hline & \multicolumn{2}{|c|}{ Stage at time of inclusion in the trial } & pT2cN0M0 G3 TCC & pT2cN0M0 G3 TCC & pT2cN0M0 G3 TCC \\
\hline & \multicolumn{2}{|c|}{ Localisation of the tumor } & right ureteral orifice & $\begin{array}{l}\text { vesico-ureteral junction } \\
\text { right }\end{array}$ & ostium left \\
\hline \multirow{6}{*}{ Treatment } & \multirow{2}{*}{$\begin{array}{l}\text { Re-TURb + PLND } \\
+ \text { implantation of } \\
\text { markers }\end{array}$} & date & $\begin{array}{l}2,5 \text { months after initial } \\
\text { diagnosis }\end{array}$ & $\begin{array}{l}\text { 1,5 month after initial } \\
\text { diagnosis }\end{array}$ & $7 / 9 / 2016$ \\
\hline & & pathology & pT0 pN0 $(0 / 20)$ cM0 & pTa pN0 $(0 / 10)$ cM0 & pT2 pN1 (1/8) cM0 \\
\hline & \multirow{4}{*}{ Radiotherapy } & date & 3 weeks after re-TURb + PLND & $\begin{array}{l}4 \text { weeks after re-TURb + } \\
\text { PLND }\end{array}$ & \multirow{4}{*}{$\begin{array}{l}\text { Patient was excluded } \\
\text { from the trial due } \\
\text { to pN1 status and } \\
\text { received neo-adjuvant } \\
\text { chemotherapy } \\
\text { followed by a radical } \\
\text { cystectomy }\end{array}$} \\
\hline & & OTT (days) & 29 days & 30 days & \\
\hline & & \multirow{2}{*}{ dose } & bladder: D50: 40 Gy (20 x 2 Gy) & $\begin{array}{l}\text { bladder: D50: } 40 \text { Gy (20 } \\
\text { x } 2 \text { Gy) }\end{array}$ & \\
\hline & & & $\begin{array}{l}\text { tumor region: D50: } 57.6 \text { Gy (20 } \\
\text { x } 2.88 \mathrm{~Gy})\end{array}$ & $\begin{array}{l}\text { tumor region: D50: } 57.6 \\
\text { Gy }(20 \times 2.88 \mathrm{~Gy})\end{array}$ & \\
\hline
\end{tabular}

Table 2: Patient's outcome and toxicity per case.

\begin{tabular}{|c|c|c|c|c|}
\hline & & & Patient 1 & Patient 2 \\
\hline \multicolumn{3}{|c|}{ Follow up (months) after diagnosis of MIBC } & 39 & 25 \\
\hline & \multirow{2}{*}{\multicolumn{2}{|c|}{ Oncological status }} & \multirow{2}{*}{$\begin{array}{l}\text { no evidence of disease on last } \\
\text { cystoscopic evaluation }\end{array}$} & $\begin{array}{l}\text { no evidence of disease on last cystoscopic } \\
\text { evaluation }\end{array}$ \\
\hline & & & & $\begin{array}{l}\text { 22/06/2017: hilar adenopathy: punction: } \\
\text { SCLC: cT1aN2M0 }\end{array}$ \\
\hline \multirow{6}{*}{ Acute toxicity } & \multirow{3}{*}{ urinary } & Grade 1 & frequency, incontinence, & nocturia \\
\hline & & Grade 2 & NR & hematuria, dysuria, incontinence, urgency \\
\hline & & Grade 3 & $\begin{array}{l}\text { nocturia (hospitalisation for } \\
\text { urosepsis based on suspicion of } \\
\text { benign prostatic hyperplasia) }\end{array}$ & frequency, retention \\
\hline & \multirow{3}{*}{$\begin{array}{c}\text { gastro- } \\
\text { intestinal }\end{array}$} & Grade 1 & frequency & $\begin{array}{l}\text { rectal blood loss, diarrhea, abdominal pain, } \\
\text { flatulence, frequency, mucus loss }\end{array}$ \\
\hline & & Grade 2 & NR & NR \\
\hline & & Grade 3 & NR & NR \\
\hline \multirow{6}{*}{ Late toxicity } & \multirow{3}{*}{ urinary } & Grade 1 & retention, dysuria & incontinence \\
\hline & & Grade 2 & nocturia & retention \\
\hline & & Grade 3 & frequency & NR \\
\hline & \multirow{3}{*}{$\begin{array}{c}\text { gastro- } \\
\text { intestinal }\end{array}$} & Grade 1 & $\begin{array}{c}\text { diarrhea, frequency, mucus loss, } \\
\text { flatulence, abdominal cramps, } \\
\text { constipation }\end{array}$ & mucus loss, urgency, frequency \\
\hline & & Grade 2 & NR & NR \\
\hline & & Grade 3 & NR & NR \\
\hline \multicolumn{5}{|c|}{$\begin{array}{c}\text { Abbreviations: TURb: Transurethral Resection of the Bladder, SCLC: Small Cell Lung Carcinoma, T: Tumor Stage, N: Nodal Stage, M: Metastases NR: not } \\
\text { reported }\end{array}$} \\
\hline
\end{tabular}
control (evaluated on cystoscopy and DW-MRI: if suspicious: histopathology), disease free survival (defined as period from start of EBRT till local or distant recurrence), cystectomy-free survival (defined as period from start of EBRT till salvage cystectomy), overall survival (defined as period from start of EBRT till death) and assessment of quality of life (EORTC QLQ-C30). 
The study was closed prematurely due to poor recruitment. Three patients were included in the study. One patient was excluded due to presence of positive lymph nodes at Pelvic Lymph Node Dissection. The patients, tumor and treatment characteristics are described in Table 1. Patient's outcome and toxicity per case are presented in Table 2. At latest follow up gastro-intestinal toxicity was limited to grade 1 constipation for the first patient and no gastro-intestinal toxicity for the second patient. Urinary toxicity at latest follow up was grade 2 nocturia and grade 1 retention for the first patient and grade 2 retention and grade 1 incontinence for the second patient.

\section{Discussion}

Proper patient selection is of utmost importance to improve the outcome of MIBC treated with BST. According to international guidelines, ideal candidates for trimodality treatment (including a TURb followed by radiochemotherapy) are patients with pT2cN0 MIBC who received a visible complete Transurethral Resection of the Bladder (TURb) and have no signs of carcinoma in situ or hydronephrosis [8-10]. In even more selected patients, chemotherapy might be omitted in order to reduce the risk of developing toxicity. Therefore, we initiated a phase 1 trial to explore the place of EBRT only with a SIB to the GTV in highly selected MIBC patients. For the latter several conditions needed to be fulfilled before patients were eligible for inclusion. First, we aimed to have a visibly complete and pathologically complete, defined as $\leq \mathrm{pT} 1$ disease, TURb by performing a re-TURb at time of insertion of the gold markers and PLND. The impact of a visibly complete resection on survival has been well recognized [11].

Additionally, we requested an ePLND as the sensitivity of current imaging in detecting involved lymph nodes is limited. By performing an ePLND, patients with occult lymph node metastasis were excluded from our trial. Moreover, retrospective surgical series have indicated that absence of pelvic lymph node involvement on ePLND (pN0), is a prognostic factor for cancer specific survival [12]. Additionally, an ePLND eradicates microscopic disease, which results in improved outcome both in $\mathrm{pN}+$ and $\mathrm{pN} 0$ disease [13]. A dose response relationship has been suggested for bladder cancer. For every additional 1 Gy a gain in 5 years OS has been suggested of $7.5 \%$ [14]. In analogy to other tumour sites one can presume that tumour recurrence is most often observed in the initial tumour region [15]. Increasing the dose to that region might result in better clinical outcome without compromising toxicity [16]. In order to define this region in case of MIBC modern imaging techniques can be used. Despite promising results regarding the role of DWMRI in local and locoregional staging of MBIC, DW-MRI is not routinely performed nowadays. For local staging DW-MRI has a sensitivity and specificity of $92.3 \%-96 \%$ and $97.6-98.1 \%[17,18]$. After transurethral resection, DW-MRI can differentiate between tumor recurrence and postoperative inflammation or fibrosis. The standard radiotherapy regimen for MIBC is irradiation of the whole bladder with a 2- to 3-cm margin to a dose of 60-66 Gy at 1.8 to $2 \mathrm{~Gy}$ fractions. However, the optimal schedule still has to be determined. Nowadays results after hypofractionated schedules are neither better nor worse than regimens of 60 Gy in 30 fractions [19].

Randomized trials comparing radiotherapy with a radiosensitizer versus radiotherapy alone have indicated a significant ad- vantage for the combined regimen in terms of disease-free survival $[4,20]$. However, patient's selection in our trial was far stricter. For example, only patients with a unique lesion were eligible. Also, the largest advantage of combined radiotherapy and a radio sensitizer has been observed in patients with more advanced stages that were excluded from our trial. Some trials have studied the place of TURb alone in MIBC. Although some long-term control has been described, the risk of having a relapse is substantial [21]. Therefore, we decided to perform a SIB to the initial tumor region as well as not to omit prophylactic irradiation of the whole bladder up to 40 Gy, which is probably enough to eradicate microscopic disease. It has also been demonstrated that a cystoscopic evaluation underestimates the extend of the disease discouraging an approach of not treating the full bladder [22].

The presented approach can also be an alternative for brachytherapy, which applies similar inclusion criteria [23]. With brachytherapy 5-year OS rates have been published that are comparable to the ones reported in surgical series [24]. Despite good clinical outcome and acceptable toxicity brachytherapy is not widely adopted due to lack of brachytherapy experience and logistic implications. The rapid dose fall-off as well as the accurate coverage of the tumor region with brachytherapy are major advantages in favour of brachytherapy compared to EBRT. With a plan-of the dayapproach the inaccuracy of patient positioning in case of bladder irradiation can be prevented. Studies describing adaptive planning, including a plan of the day approach, reported increased coverage of the bladder and tumor with reduction of the doses delivered to the surrounding organs at risk [25].

Also, the implementation of modern radiation techniques such as intensity-modulated arc therapy allows escalating the dose to the initial tumor region while sparing intestinal loops and rectal mucosa [26-27]. Unfortunately, our study was closed prematurely due to poor accrual. Conclusions on maximum tolerated dose can therefore not be drawn from our study. Nevertheless, the few patients that were included in our trial did not develop major toxicity and oncological outcome is excellent with more than 2 years of follow up. Therefore, our study might be considered as hypothesis generating for developing studies with high dose EBRT in well-selected MIBC patients.

\section{References}

1. Novara G, Catto JW, Wilson T, Annerstedt M, Chan K, et al. (2015) Systematic review and cumulative analysis of perioperative outcomes and complications after robot-assisted radical cystectomy. Eur Urol 67: 376-401.

2. Zhong J, Switchenko J, Jegadeesh NK, Cassidy RJ, Gillespie TW, et al. (2018) Comparison of outcomes in patients with muscle-invaisve bladder cancer treated with radical cystectomy versus bladder preservation. Am J Clin Oncol 42(1): 36-41.

3. Kulkarni GS, Hermanns T, Wei Y, Bhindi B, Satkunasivam R, et al. (2017) Propensity score analysis of radical cystectomy versus bladder-sparing trimodal therapy in the setting of a multidisciplinary bladder cancer clinic. J Clin Oncol 35: 2299-2305.

4. James ND, Hussain SA, Hall E, Peter Jenkins, Jean Tremlett, et al. (2012) Radiotherapy with or without chemotherapy in muscle-invasive bladder cancer. N Engl J Med 366: 1477-1488.

5. Pos Fj, Hart G, Schneider C, Sminia P (2006) Radical radiotherapy for invasive bladder cancer: what dose and fractionation schedule to choose? Int J Radiat Oncol Biol Phys 2006; 64: 1168-1173. 
6. Bellmunt J, Orsola A, Leow JJ, Wiegel T, De Santis M et al. (2014) Bladder cancer: ESMO practice guidelines for diagnosis, treatment and follow up. Ann Oncol 25: 40-48.

7. Witjes JA, Lebret, T, Compérat EM, Cowan NC, De Santis M, et al. (2017) Updated 2016 EAU guidelines on muscle-invasive and metastatic bladder cancer. Eur Urol 71: 462-475.

8. Spiess PE, Aqarwal N, Bangs R, Boorjian SA, Buyyounouski MK, et al (2017) Bladder cancer, version 5.2107, NCCN clinical practice guidelines in oncology. J Natl Compr Can Netw 15: 1240-1267.

9. Fonteyne V, Ost P, Vanpachtenbeke F, Colman R, Sadeghi S, et al. (2014) Rectal toxicity after intensitity modulated radiotherapy for prostate cancer: which rectal dose volume constraints should we use? Radiother Oncol 113: 398-403.

10. Chang SS, Bochner BH, Chou, Dreicer R, Kamat AM, et al. (2017) Treatment of non-metastatic muscle-invasive bladder cancer: AUA/ ASCO/ASTRO/SUO Guideline. J Urol 198: 552-559.

11. Giacalone NJ, Shipley WU, Clayman RH, Niemierko A, Drumm M, et al. (2017) Long-term outcomes after bladder-preserving tri-modality therapy for patients with muscle-invasive bladder cancer: an updated analysis of the Massachusetts General Hospital Experience. Eur Urol 71: 952-960.

12. Bruins HM, Veskimae E, Hernandez V, Imamura M, Neuberger MM, et al. (2014) The impact of the extent of lymphadenectomy on oncologic outcomes in patients undergoing radical cystectomy for bladder cancer: a systematic review. Eur Urol 66(6): 1065-1077.

13. Brunocilla E, Pernetti R, Schiavina R, Borghesi M, Vagnoni V, et al. (2013) The number of nodes removed as well as the template of the dissection is independently correlated to cancer-specific survival after radical cystectomy for muscle-invaisve bladder cancer. Int Urol Nephrol 45 711-719.

14. Majewski W, Maciejewski B, Majewski S, Suwinski R, Miszczyk L, et al. 2004) Clinical radiobiology of stage T2-T3 bladder cancer. Int J Radiat Oncol Biol Phys 60: 60-70.

15. Pucar D, Hricak H, Shukla Dave A, Kuroiwa K, Drobnjak M, et al. (2007) Clinically significant prostate cancer local recurrence after radiation therapy occurs at the site of primary tumor: magnetic resonance imaging and step-section pathology evidence. Int J Radiat Oncol Biol Phys 69: 62-69.

16. Monninkhof EM, Van Loon JWL, Van Vulpen M, Kerkmeijer LGW, Pos FJ, et al. (2018) Standard whole prostate gland radiotherapy with and without lesion boost in prostate cancer: Toxicity in the FLAME randomized controlled trial. Radiother Oncol 127: 74-80.

\section{ISSN: 2574-1241}

DOI: 10.26717/BJSTR.2019.17.002956

Valérie Fonteyne. Biomed J Sci \& Tech Res

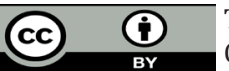

This work is licensed under Creative

Commons Attribution 4.0 License

Submission Link: https://biomedres.us/submit-manuscript.php
17. Giannarini g, Giuseppe P, Harriet CT (2012) Potential and limitations of diffusion-weighted magnetic resonance imaging in kidney, prostate and bladder cancer including pelvic lymph node staging: a critical analysis of the literature. Eur Urol 61: 326-340.

18. El Assmy A, Abou El Ghar M, Refaie HF, Mosbah A, El Diasty T (2012) Diffusion-weighted magnetic resonance imaging in follow-up of superficial urinary bladder carcinoma after transurethral resection: initial experience. BJU International 110: 622-627.

19. Plataniotis GA, Dale RG (2013) Radio-chemotherapy for bladder cancer: contribution of chemotherapy on local control. World J Radiol 5: 267274.

20. Hoskin PJ, Rojas AM, Bentzen SM, Saunders MI (2010) Radiotherapy with concurrent carbogen and nicotinamide in bladder carcinoma. J Clin Oncol 28: 4912-4918.

21. Solsona E, Iborra I, Collado A, Rubio Briones J, Casanova J, et al. (2010) Feasibility of radical transurethral resection as monotherapy for selected patients with muscle invasive bladder cancer. J Urol 184(2): 475-480.

22. Goldsmith B, Tucker K, Conway RG, He J, Guzzo T, et al. (2013) Discordance between preoperative and postoperative bladder location: implications for partial-bladder radiation. Int J Radiat Oncol Biol Phys 85: 707-713.

23. Pieters BR, Van der Steen Banasik E, Smits GA, De Brabandere M, Bossi A, et al. (2017) GEC-EWTRO/ACROP recommendation for performing bladder sparing treatment with brachytherapy for muscle-invasive bladder cancer. Radiother Oncol 122: 340-346.

24. Koning CC, Blank LE, Koedooder C, RM Van Os, M Van De Kar, et al. (2012) Brachytherapy after external beam radiotherapy and limited surgery preserves bladders for patients with solitary pT1-pT3 bladder tumors. Ann Oncol 23: 2948-2953.

25. Webster GJ, Stratford J, Rodgers J, Livsey JE, Macintosh D, et al. (2013) Comparison of adaptive radiotherapy techniques for the treatment of bladder cancer. Br J Radiol 86(1021): 20120433.

26. Fonteyne V, Lumen N, Ost P, Van Praet C, Vandecasteele K, et al. (2013) Hypofractionated intensity-modulated arc therapy for lymph node metastasized prostate cancer: toxicity and 3-years clinical outcome. Radiother Oncol 109: 229-234.

27. Vandecasteele K, De Neve W, De Gersem W, Delrue L, Paelinck L, et al (2009) Intensity-modulated arc therapy with simultaneous integrated boost in the treatment of primary irresectable cervical cancer. Treatment planning, quality control and clinical implementation. Strahlenther Onkol 185: 799-807.

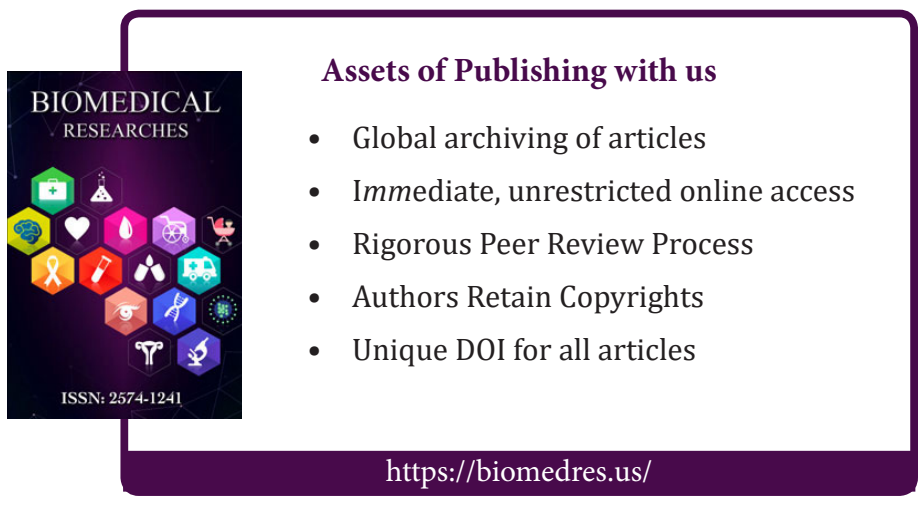

